\title{
Sử dụng đồng thời quan trắc quy mô lớn và quy mô bão trong việc tăng cường thông tin ban đầu cho bài toán dự báo xoáy thuận nhiệt đới bằng mô hình số trị
}

\author{
Dư Đức Tiến ${ }^{1, *}$, Ngô Đức Thành², Kiều Quốc Chánh ${ }^{3}$ \\ ${ }^{I}$ Trung tâm Dư báo khí tương thủy văn Trung ưong \\ ${ }^{2}$ Truờng đại học Khoa học và Công nghệ Hà Nọi, \\ Viện Hàn lâm Khoa học và Công nghệ Việt Nam \\ ${ }^{3}$ Đại hoc Indiana, Hoa Kỳ
}

Nhận ngày 08 tháng 8 năm 2016

Chỉnh sửa ngày 26 tháng 8 năm 2016; Chấp nhận đăng ngày 16 tháng 12 năm 2016

\begin{abstract}
Tóm tắt: Sử dụng đồng thời thông tin quan trắc hòa hợp từ quy mô lớn đến quy mô bão, bài báo đưa ra một phương pháp tăng cường thông tin ban đầu một cách khách quan cho mô hình dự báo số trị khu vực WRF-ARW của NCEP (Trung tâm nghiên cứu môi trường quốc gia, Mỹ) ứng dụng trong dự báo bão bằng phương pháp đồng hóa tổ hợp lọc Kalman (LETKF). Thông tin quy mô bão được tạo ra dựa trên việc xây dựng mô hình xoáy 3 chiều đầy đủ từ thông tin phân tích bão thực tế trong nghiệp vụ của NCEP. Thông tin quan trắc quy mô lớn là số liệu gió tại các mực trên cao tính toán từ sự dịch chuyển của mây do trung tâm CIMSS (Trường đại học Wisconsin, Mỹ) cung cấp. Các dẫn giải về phương pháp sẽ được giới thiệu cùng một số kết quả thử nghiệm ban đầu với trường hợp cơn bão Usagi năm 2013 hoạt động trên khu vực Tây Bắc Thái Bình Dương (TBTBD). Một số kết quả bước đầu cho thấy tính hiệu quả của phương pháp đồng hóa đồng thời các thông tin ở các quy mô khác nhau trong dự báo quỹ đạo và cường độ của cơn bão.
\end{abstract}

Tù khóa: Ban đầu hóa bão, đồng hóa tổ hợp, cấu trúc bão 3 chiều.

\section{Bài toán tăng cường cấu trúc xoáy ban đầu cho mô hình số trong dự báo bão}

Một trong những nguyên nhân gây ra sai số dự báo bão, đặc biệt đến cường độ bão là cấu trúc ban đầu của bão chưa được mô tả tốt cho các mô hình số trị do sự thiếu hụt quan trắc trên biển. Khi đó các thông tin sử dụng trong việc phân tích trường ban đầu đặc biệt liên quan đến các hoàn lưu quanh khu vực tâm bão cho các

\footnotetext{
*Tác giả liên hệ. ĐT.: 84-936067015

Email: duductien@gmail.com
}

mô hình số sẽ hết sức hạn chế, các thông tin quy mô vừa và quy mô đối lưu của các đặc trưng ban đầu của bão sẽ bị làm trơn đi, dẫn tới các kết quả tích phân sẽ không phản ánh được đúng những diễn biến của hiện tượng này [8]. Để khắc phục hạn chế thiếu hụt quan trắc trên biển, bên cạnh các quan trắc từ số liệu vệ tinh địa tĩnh những năm 1950-1960, từ những năm 1980, các thám sát thẳng đứng từ các đầu đo được mang trên các vệ tinh cực đã được phát triển, điển hình nhất và vẫn sử dụng đến thời điểm hiện tại là các số liệu AMSU và HIRS để đo đạc các profile thẳng đứng liên quan đến 
nhiệt độ và độ ẩm của khí quyển [13]. Ngoài ra, từ số liệu của vệ tinh địa tĩnh cho phép chiết suất đưa vào mô hình trường gió trên cao thông qua việc xác định sự dịch chuyển của các phần tử mây mỏng hoặc tựa trong suốt giữa các khoảng thời gian liên tiếp nhau [10]. Mặc dù đã có sự bổ sung cấu trúc khí quyển trên biển từ số liệu vệ tinh, tuy nhiên các đặc trưng động lực ba chiều phân giải cao vẫn hết sức hạn chế để có thể đáp ứng được bài toán mô phỏng và dự báo xoáy thuận nhiệt đới/bão với quy mô không gian ngang dưới $10 \mathrm{~km}$. Riêng dữ liệu về bão từ các thám sát sử dụng máy bay bay vào bão và đo đạc (dropsonde) mặc dù có chất lượng thông tin cao nhưng chi phí hết sức đắt đỏ và do đó rất hạn chế trong công tác nghiệp vụ.

Nhận thức được vai trò quan trọng của cấu trúc nhiệt-động lực của bão ban đầu đến kết quả mô phỏng và dự báo bão và do bản thân sự thiếu hụt quan trắc đã dẫn tới các phân tích trường qui mô lớn thường quá yếu so với thực tế [8], lớp bài toán ban đầu hóa xoáy (Tropical Cyclone Initialization) đã ra đời với nguyên tắc chính là đưa được vào thông tin xoáy có cấu trúc thật hơn thay thế cấu trúc xoáy không thật do các quan trắc hiện tại chưa đảm bảo việc thể hiện cấu trúc thật của bão trong trường ban đầu. Phương pháp cài xoáy lý tưởng vào trường ban đầu của mô hình hoặc các xoáy lý tưởng (bogus vortex, model vortex, synthetic vortex) được gọi chung là bogus. Các xoáy lý tưởng được xây dựng dựa trên các thông tin quan trắc về bão, bao gồm vị trí có áp suất mực biển cực tiểu, tốc độ gió bề mặt cực đại Vmax, áp suất mực biển cực tiểu Pmin, bán kính gió cực đại, bán kính gió $15 \mathrm{kts}$ hoặc $30 \mathrm{kts}$. Hiện nay có 3 lớp phương pháp ban đầu hóa xoáy bão chính gồm: i) phương pháp bogus thực nghiệm, ii) phương pháp ban đầu hóa động lực và iii) phương pháp bogus khách quan.

\subsection{Phuoong pháp bogus thực nghiệm}

Phương pháp bogus thực nghiệm giả thiết xoáy ban đầu là một xoáy nhiễu trên trường quy mô lớn và cần phải thay thế xoáy nhiễu này bởi một xoáy có cấu trúc phù hợp nhất (bogus lý tưởng) với các đặc điểm chính của bão thông qua các quan trắc ước lượng về bão như cường độ, ví trị, bán kính gió mạnh nhất,... Quá trình thay thế xoáy nhiễu bằng xoáy lý tưởng phải được thực hiện một cách hòa hợp, tránh sự mất cân bằng giữa các trường và sự phát sinh nhiễu, gia tăng biên độ nhiễu trong quá trình tích phân của mô hình. Ba đặc điểm chính cần phải đảm bảo trong một cấu trúc xoáy lý tưởng bao gồm: i) sự thay đồi theo khoảng cách tính từ tâm bão của profile gió tiếp tuyến và áp suất, ii) sự thay đổi theo độ cao của gió và áp suất và iii) xem xét đến tính đối xứng và bất đối xứng của bão. Hai phân bố đặc trưng nhất cho xoáy bão gồm: i) phân bố trường khí áp suất với giá trị cực tiểu tại tâm bão và tăng dần theo khoảng cách so với tâm bão và ii) phân bố gió tiếp tuyến với giá trị cực tiểu tại tâm bão và mạnh dần theo khoảng cách đến giá trị bán kính tốc độ gió cực đại rồi tiếp tục giảm dần theo khoảng cách so với tâm bão. Các profile gió tiếp tuyến trong những nghiên cứu hiện nay về cơ bản đều dựa trên profile gió tiếp tuyến của xoáy trong cơ học chất lỏng nhớt do William John Macquorn Rankine tìm ra ở thế kỉ 19 [4], gọi tắt là các profile Rankine, có dạng:

$$
V(r)= \begin{cases}V_{m} \frac{r}{r_{m}} & r<r_{m} \\ V_{m}\left(\frac{r}{r_{m}}\right)^{-1} & r \geq r_{m}\end{cases}
$$

trong đó $V(r)$ là gió tiếp tuyến tại khoảng cách $r$ so với tâm xoáy, $V_{m}$ là tốc độ gió tiếp tuyến cực đại và $r_{m}$ là bán kính gió cực đại. Hạn chế chính của profile Rankine là không khả vi tại vị trí bán kính gió cực đại và không mô tả được hoàn lưu phía ngoài bão. Hai công thức điển hình miêu tả profile của gió tiếp tuyến và áp suất cải tiến từ dạng profile Rankine gồm trong nghiên cứu của Fujita (1952) và Kurihara (1993) [4, 8]. Trong Fujita (1952), phân bố áp suất bề mặt có dạng:

$$
P(r)=P_{E}-\Delta P\left[1+\left(\frac{r}{R_{0}}\right)^{2}\right]^{-1 / 2}
$$


trong đó $r$ là bán kính, $P_{E}$ là áp suất bề mặt môi trường, $\Delta P$ đặc trưng cho cường độ xoáy và $R_{0}$ đặc trưng cho kích thước xoáy. Công thức phân bố áp suất (2) được mở rộng và ứng dụng trong rất nhiều công trình, ví dụ $[9,14]$. Đối với phân bố gió tiếp tuyến $V(r, \sigma, t)$ theo bán kính $r$, mực mô hình $\sigma$ và thời gian $t$, trong Kurihara (1993) có dạng:

$$
V(r, \sigma, t)=<V>(r, \sigma) \exp \left(1-\frac{\tau}{t}\right)
$$

trong đó phụ thuộc vào quy mô thời gian điều chỉnh $\tau,<\mathrm{V}>$ là phân bố gió mục tiêu cho trước. Công thức (3) thường được mở rộng và ứng dụng trong một số mô hình chính áp. Một bước quan trọng của phương pháp bogus thực nghiệm là quá trình loại bỏ trường xoáy nhiễu ra khỏi trường ban đầu để giữ lại trường quy mô lớn. Phương pháp loại bỏ đơn giản có thể được thực hiện bằng cách áp dụng các toán tử lọc, làm trơn theo không gian cho các trường áp suất (hoặc địa thế vị) hoặc có thể áp dụng phương pháp phân tích chuỗi Fourie và giữ lại thành phần sóng chính [8].

\subsection{Phương pháp ban đầu hóa động lực}

Tương tự phương pháp bogus thực nghiệm với việc sử dụng các profile cân bằng thực nghiệm cho cấu trúc xoáy bão, tuy nhiên điểm khác biệt cơ bản của phương pháp động lực là sử dụng chính mô hình để tạo sự cân bằng và sinh ra xoáy nhân tạo phù hợp nhất so với quan trắc, các xoáy này còn gọi là các xoáy bogus tự mô hình (model self bogus vortex), sơ đồ điển hình hiện nay như nghiên cứu của Nguyen và Chen (2011) [9]. Trên thực tế đây là phương pháp khá phức tạp nhưng giảm thiểu được việc bất cân bằng động lực trong phương pháp bogus thực nghiệm. Ví dụ trong nghiên cứu [9] với việc mô phỏng lại cơn bão Morakot, cấu trúc ban đầu được thực hiện bằng cách chạy lặp một số lần hữu hạn (80 lần) mô hình khu vực WRF với thời hạn tích phân ban đầu là $1 \mathrm{~h}$. Các lần lặp sau lấy kết quả của lần lặp trước làm điều kiện ban đầu để tích phân lại. Quá trình chạy lặp này cho phép xoáy ban đầu cân bằng động lực với trường quy mô lớn ban đầu cho trước.

\subsection{Phưong pháp bogus khách quan}

Dựa trên đặc tính của phương pháp đồng hóa biến phân là phân tích ước lượng trạng thái tối ưu có khả năng xảy ra cao nhất ứng với một tập quan trắc và một trạng thái nền cho trường, Zou và Xiao (2000) đã đề xuất ứng dụng phương pháp này trong bài toán ban đầu hóa xoáy [14]. Tư tưởng chính của phương pháp là sau khi xây dựng xoáy giả lý tưởng, việc đưa thông tin các xoáy giả này vào mô hình phải được thực hiện một cách khách quan không giống như khâu thực hiện thứ 2 trong các phương pháp bogus thực nghiệm thực hiện là thông qua các quan hệ như cân bằng phi tuyến phía trên lớp biên, quan hệ gió gradient, quan hệ địa chuyển, cân bằng thũy tĩnh... để tính toán cấu trúc lại từng biến của mô hình. Các quá trình này hết sức phức tạp và phải thiết lập lại phụ thuộc vào từng cấu trúc của các mô hình khác nhau. Nếu xem xoáy giả như là một quan trắc cần đồng hóa vào (thông qua đặc tính động lực rõ rệt nhất đặc trưng cho bão bằng các profile gió và áp suất), quá trình đồng hóa sẽ phân tích tối ưu lại trường ban đầu giữa trên các thông tin quan trắc lý tưởng này trên toàn miền tính mà không mang tính áp đặt như các phương pháp bogus thực nghiệm. Tác giả cũng mong muốn đây là phương pháp tổng quát hóa bài toán ban đầu hóa xoáy cho tất cả các mô hình - điều mà các phương pháp bogus thực nghiệm không đạt được. Tuy nhiên trên thực tế một hạn chế ở đây chính là bản thân việc xây dựng hệ thống đồng hóa số liệu cũng phụ thuộc cho từng mô hình, việc xây dựng hệ thống đồng hóa có thể còn phức tạp hơn cả các phương pháp bogus thực nghiệm. Ngoài ra cũng cần thiết đưa ra được ma trận sai số hiệp biến phương sai nền $\mathrm{B}$ như là điều kiện cần trong phương pháp đồng hóa biến phân. Những điểm hạn chế này cũng phần nào làm giảm khả năng tổng quát hóa phương pháp cài xoáy theo đề xuất của Zou. 
Với ý tưởng từ nghiên cứu của Zou và Xiao (2000), Phan Văn Tân và cộng sự (2009) đã sử dụng hệ thống WRFDA để đồng hóa thông tin xoáy giả trên một số mực mô hình, trong đó đối với bão mạnh số liệu giả được trích ra trên 3 mực $1000 \mathrm{mb}, 850 \mathrm{mb}$ và $700 \mathrm{mb}$ của xoáy nhân tạo, trong khi với bão yếu thì chỉ được trích dẫn trên hai mực $1000 \mathrm{mb}$ và $850 \mathrm{mb}$ để làm profile quan trắc đưa vào đồng hóa [2]. Xoáy giả được xây dựng theo nghiên cứu của Roger Smith dựa trên lý thuyết cân bằng ba chiều. Với tập mẫu hạn chế (10 cơn bão trên khu vực Biển Đông) và mới chỉ bước đầu đánh giá những hiệu ứng ảnh hưởng sai số quỹ đạo của các cơn bão hạn dự báo đến $72 \mathrm{~h}$ như đối với các cơn bão tương đối mạnh, quĩ đạo dự báo đã được cải thiện, còn đối với các cơn bão yếu, các thử nghiệm cho thấy ít có hiệu ứng đối với quỹ đạo dự báo.

Với hạn chế chính của phương pháp đồng hóa biến phân liên quan đến việc phải định ra ma trận sai số hiệp biến trường nền trong thủ tục cực tiểu hóa và nhằm giảm được tính bất định trong dự báo, các nghiên cứu gần đây đã cập nhật theo hướng sử dụng phương pháp đồng hóa tổ hợp. Điển hình nhất là công trình của Wang và cộng sự (2014) với việc tập trung vào việc tăng cường thông tin cấu trúc bão bằng số liệu thám sát mật độ cao radar thông qua phương pháp đồng hóa tổ hợp sử dụng bộ lọc Kalman [12]. Như vậy, áp dụng phương pháp đồng hóa biến phân và đồng hóa tổ hợp trong việc tăng cường thông thông tin xoáy bão ban đầu cho các mô hình là môt bước phát triển so với lớp phương pháp cài xoáy thực nghiệm được phát triển trong những năm 1990 thế kỉ trước.

Về mức độ linh hoạt, rõ ràng có thể thấy phương pháp bogus khách quan ngoài việc vẫn có thể đưa các thông tin xoáy lý tưởng giống phương pháp thực nghiệm vào mà còn có thể đồng hóa được các quan trắc mới đặc trưng được cho bão chỉ thông quan việc xây dựng toán tử quan trắc - độc lập với mô hình. So với phương pháp đồng hóa biến phân thì phương pháp đồng hóa tổ hợp sử dụng bộ lọc Kalman (EnKF) đã cho phép phần nào tổng quát hóa thực sự bài toán ban đầu hóa xoáy bão cho mô hình số trị. Kalnay và cộng sự (2007) đã chỉ ra rằng EnKF có thể so sánh được với hệ thống 4DVAR tuy nhiên việc thuận tiện trong các tham số quan trắc mới đưa vào như hai tham số vị trí và tốc độ dịch chuyển của bão là không đơn giản khi áp dụng vào một hệ 4DVAR [6]. Điều này đặc biệt quan trọng khi mà các thám sát với các hình thức khác nhau được phát triển để quan trắc và giám sát bão một cách phi truyền thống có thể được tận dụng nhanh khi áp dụng phương pháp EnKF.

Dựa trên những điểm mạnh của phương pháp đồng hóa tổ hợp, trong nghiên cứu sẽ tăng cường cấu trúc của xoáy bão cho mô hình dựa trên phương pháp LETKF. Để làm được việc này, hệ thống chương trình xử lý số liệu phân tích bão thực và chương trình xây dựng một xoáy nhân tạo ba chiều đầy đủ đã được thực hiện (phần 2). Các thông tin xoáy nhân tạo này sẽ được đồng hóa đồng thời (blending) với các quan trắc quy mô lớn khác (gió vệ tinh) thông qua phương pháp LETKF (phần 3).

\section{Phương pháp tạo cấu trúc xoáy ba chiều nhân tạo từ thông tin quan trắc bão thực}

\subsection{Co sở lý thuyết và tính toán module mô phỏng xoáy bão nhân tạo}

Trước hết, sự thay đổi của gió theo độ cao được lấy từ một nghiệm tại thời điểm ban đầu $(\mathrm{t}=0)$ trong mô hình giải tích đầy đủ do Kieu và Zhang (2009) thiết lập [7]. Công thức đầy đủ cho cấu trúc gió tiếp tuyến bão được đưa ra theo công thức:

$$
V(r, \sigma)=V_{m} \frac{r}{r_{m}} \sin \left(\frac{\pi}{2} \sigma\right)^{1-\delta} \cos \left(\frac{\pi}{2} \sigma\right)^{\delta} \exp \left\{\frac{1}{b}\left[1-\left(\frac{r}{r_{m}}\right)^{b}\right]\right\}
$$


trong đó $V_{m}$ là gió cực đại, $r_{m}$ bán kính gió cực đại, $r$ là bán kính tính từ tâm bão, $\sigma$ là mực mô hình, $b$ là tham số xác định dạng phân bố ngang của profile gió (được đặt bằng 0.7 theo nghiên cứu của hệ thống dự báo bão nghiệp cụ HWRF của NCEP, tham khảo báo cáo của Tallapragada và cộng sự năm 2014), $\delta$ là tham số tự do thay đổi từ $[0,1]$ để xác định độ cao của vị trí đạt giá trị gió bề mặt cực đại $(\delta=0$ tương đương với gió cực đại tại bề mặt và ( $\delta=1$ ứng với gió cực đại tại mực giữa) và là độ cao so với bề mặt (mực mô hình) [11].

Với phân bố gió trên, nhiễu động địa thế vị sẽ được tính toán tiếp theo bằng cách giải lặp hệ phương trình cân bằng phi tuyến cho gió sau (theo Holton (2004), trang 390 [5], ký hiệu cho cho toán tử gradient và $\mathcal{J}$ cho toán tử Jacobian):

$$
\boldsymbol{\nabla}^{2} \Phi=\boldsymbol{\nabla} \cdot(f \nabla \psi)-\mathcal{J}\left(\frac{\partial \psi}{\partial x}, \frac{\partial \psi}{\partial y}\right)
$$

Để đơn giản hóa và giảm thời gian tính toán giải lặp phương trình Poison cho biến địa thế vị, chúng tôi áp dụng phương pháp lặp Lipman 2 chiều trên từng mực độ cao khác nhau, chi tiết về giải phương trình Poison và phương pháp lặp Lipman [3]. Cụ thể thực hiện như sau:

Buớc 1: Thực hiện vòng lặp toàn bộ miền tính giả định $\boldsymbol{T}$ chỉ bao phủ vùng xoáy bão và thực hiện: Xác định bán kính $r$ trong công thức (4) từ tâm bão đến điểm lướii, $j, k$. Tính giá trị gió tiếp tuyến theo công thức (4) và chuyển đổi sang giá trị thành phần gió kinh hướng $(\mathrm{u})$ và vĩ hướng $(\mathrm{v})$ tại điểm $i, j, k$

Bước 2: Trường hàm dòng $\psi$ và toán tử Jacobian từ trường gió $\mathrm{u}, \mathrm{v}$ đã xác định ở bước 1 được áp dụng theo công thức sai phân hữu hạn trung tâm, tại các vùng biên áp dụng sai phân tiến và lùi. Toàn bộ vể phải của công thức (5) ký hiệu lại làrhs $(i, i, k)$ ứng với giá trị tại từng điểm lưới trên miền tính $\boldsymbol{T}$

Bước 3: Giải lặp phương trình (5) trên từng mực miền tính theo phương pháp Lipman với số lần lặp hữu hạn, khi đó bước lặp thứ $(n+1)$ cho $\Phi$ sẽ có dạng:

$$
\Phi_{i, j, k}^{(n+1)}=\frac{\left(\Phi_{i-1, j, k}^{(n)}+\Phi_{i+1, j, k}^{(n)}\right) \Delta y^{2}+\left(\Phi_{i, j+1, k}^{(n)}+\Phi_{i, j-1, k}^{(n)}\right) \Delta x^{2}-\Delta x^{2} \Delta y^{2} r h s(i, j, k)}{2\left(\Delta x^{2}+\Delta y^{2}\right)}
$$

Sai số giữa bước thứ $n$ và $n+1$ chênh nhau dưới $1 \%$ thì phép lặp được dừng lại. Sau khi tính được giá trị địa thế hoặc nhiễu động nhiệt độ thế vị tại từng lớp khí quyển, ta xác định tương ứng nhiệt độ/nhiễu độ nhiệt độ tại từng lớp khí quyển theo phương trình cân bằng thủy tĩnh $\left(\frac{\partial p}{\partial z}=\right.$ $-\rho g$ ), phương trình trạng thái cho mật độ khí quyển $(\rho=p R T)$ và công thức chuyển đổi độ cao địa thế vị và độ cao $(d \Phi=g d z)$.

Trong hình 1 minh họa kết quả tính toán cụ thể xoáy nhân tạo cho cơn bão Usagi tại thời điểm 2013-09-19 lúc 00UTC, lưới tính $\boldsymbol{T}$ có độ phân giải $12 \mathrm{~km}$ x $12 \mathrm{~km}$ x 31 mực của mô hình WRF-ARW, các tham số phát báo từ Trung tâm cảnh báo bão của Mỹ JTWC gồm tâm tại $16.9 \mathrm{~N} ; 128.5 \mathrm{E}$, sức gió mạnh nhất Vmax đạt 48 $\mathrm{m} / \mathrm{s}$ với bán kính rmax đạt $37 \mathrm{~km}$ và áp suất cực tiểu tại tâm đạt $952 \mathrm{hPa}$. Trong hình 1e cung cấp số liệu ước lượng gió bề mặt mực $10 \mathrm{~m}$ từ vệ tinh cực của NOAA (Trung tâm quản trị khí quyển và đại dương quốc gia, Mỹ) tại cùng thời điểm mô phỏng xoáy nhân tạo cho cơn bão Usagi cho thấy phân bố gió (hình 1a) phù hợp với phân bố ước lượng này.

Ngoài ra, trường nhiệt độ thế vị (hình 1c) cũng mô phỏng rõ được cấu trúc lõi nóng tầng đối lưu của bão. Đánh giá sơ bộ với trường gió xoáy của mô hình toàn cầu GFS làm trường ban đầu cho mô hình WRF-ARW (hình 1f) thấy rằng so với tâm quan trắc, GFS có xu thế lệch về phía trái so với thực tế. Ngoài ra, cường độ đánh giá qua gió bề mặt cực đại VMAX phân tích ban đầu của GFS đạt $35 \mathrm{~m} / \mathrm{s}$ so với thực tế là $48 \mathrm{~m} / \mathrm{s}$ còn VMAX trong xoáy nhân tạo đạt $45 \mathrm{~m} / \mathrm{s}$. Như vậy, với thông tin VMAX và vị trí thật hơn trong xoáy nhân tạo 3 chiều so với GFS sẽ là thông tin bổ sung hữu ích cho trường ban đầu của mô hình. 
(a)

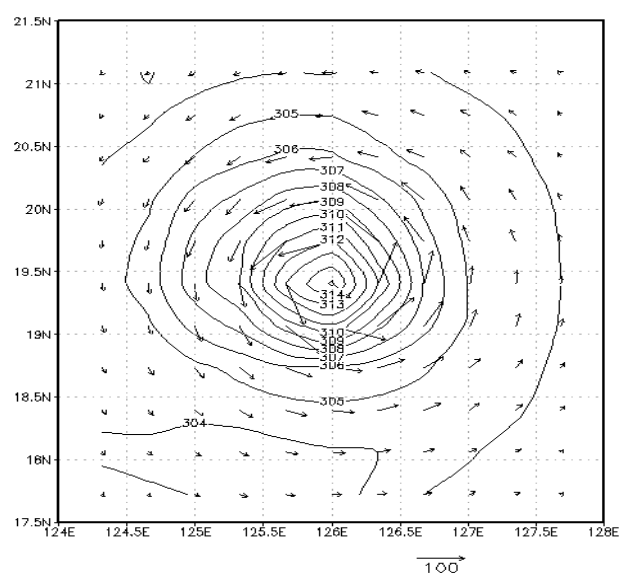

(c)

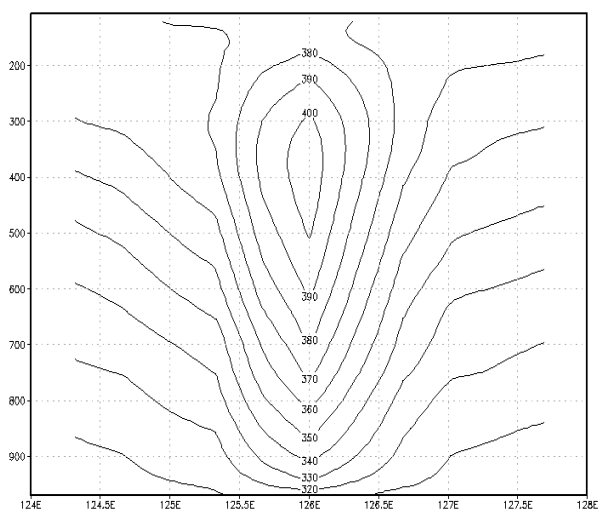

(e)

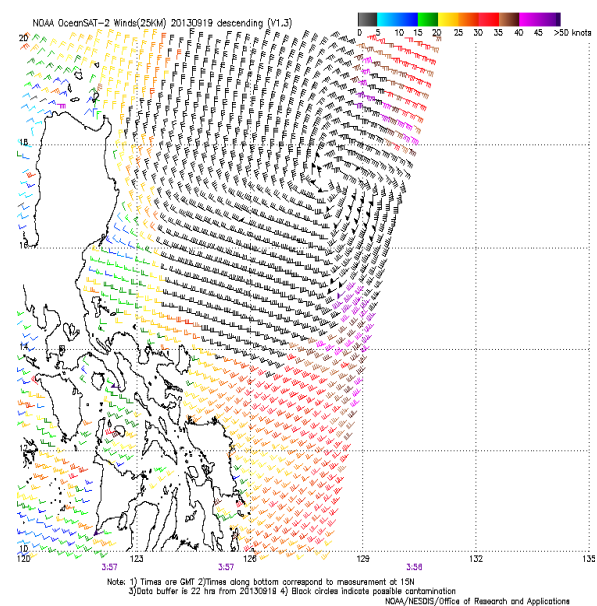

(b)

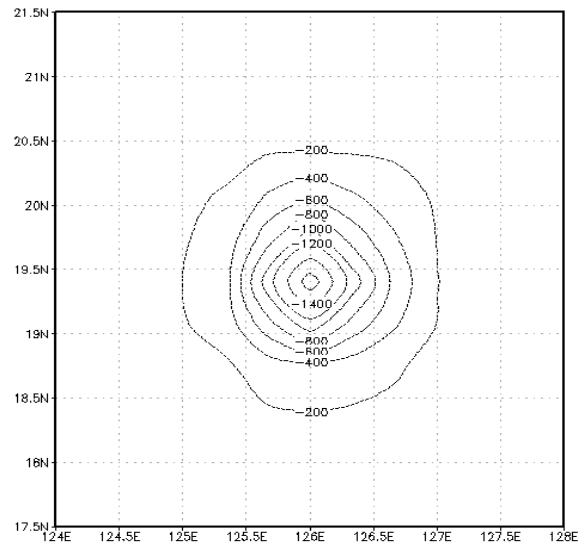

(d)

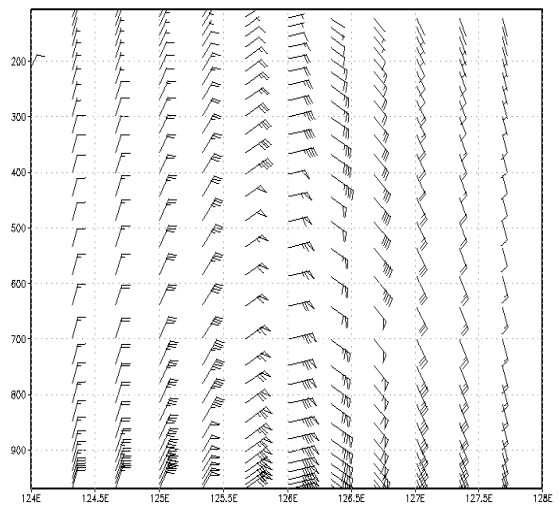

(f)

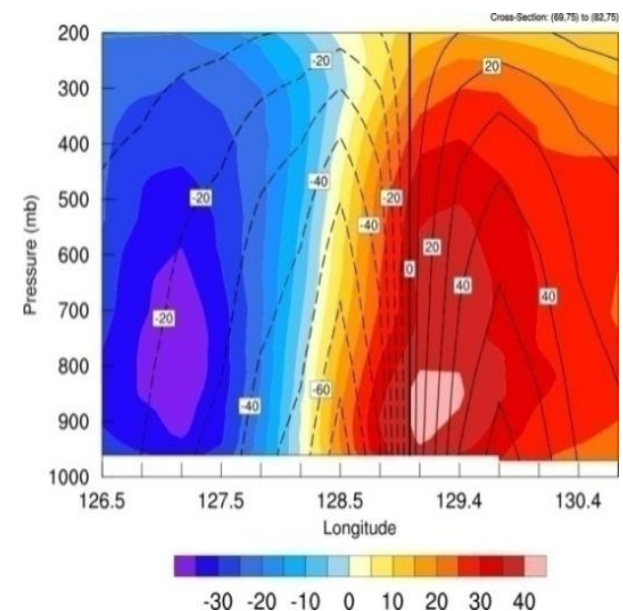

Hình 1. (a) là phân bố gió và nhiệt độ thế vị [K] ở mực thấp nhất, (b) nhiễu động địa thế vị [Pa] mực thấp nhất, (c) là mặt cắt thẳng đứng của nhiệt độ thế vị $[\mathrm{K}]$ tại tâm cơn bão, (d) là mặt cắt thẳng đứng tại tâm bão của trường gió [kts], (e) gió quan trắc vệ tinh mực $10 \mathrm{~m}[\mathrm{kts}]$, (f) mặt cắt tại tâm bão thành phần gió tiếp tuyến nhân tạo (đường đẳng trị) và trường ban đầu của mô hình GFS (đường đẳng trị có tô màu), đơn vị $[\mathrm{m} / \mathrm{s}]$. 


\subsection{Thiết lập quan trắc hòa hợp giữa thông tin quy mô lớn và xoáy nhân tạo}

Số liệu quan trắc quy mô lớn được sử dụng để bổ sung đồng hóa vào mô hình được lấy từ dữ liệu gió dịch chuyển AMV của CIMSS (gọi tắt là $\mathrm{AMV}$ ). Trên thực tế mặc dù số liệu $\mathrm{AMV}$ đã được đồng hóa cho mô hình GFS, tuy nhiên theo nhiều đánh giá cho thấy quá trình nội suy từ lưới toàn cầu xuống lưới quy mô vừa làm điều kiện ban đầu và điều kiện biên phần nào đã là đi khá nhiều những thông tin quan trắc này và quá trình tái đồng hóa các thông tin quan trắc này vẫn cho phép tái cung cấp được các thông tin quan trắc đến trường phân tích của các hệ mô hình quy mô khu vực. Số liệu gió AMV được cung cấp tại địa chỉ lưu trữ của CIMSS:
http://www.tropic.ssec.wisc.edu/archive/data/N WPacific/. Để làm đầu vào cho mô hình xoáy 3 chiều nhân tạo trong mục 2.1, các quan trắc bão thật được lấy từ những phân tích thời gian thực của JTWC theo dạng mẫu file TCVital bao gồm thông tin bán kính gió cực đại, tốc độ gió cực đại bề mặt và vị trí của cơn bão. NCEP cũng cung cấp số liệu TCVital tại địa chỉ trực tuyến của mô hình GFS: http://www.ftp.ncep.noaa. gov/data/nccf/com/gfs/prod.

Hình 2 minh họa mặt cắt ngang tại mực $195 \mathrm{hPa}$ và mặt cắt tại kinh tuyến $130 \mathrm{E}$ của quan trắc hòa hợp hai trường quy mô lớn và xoáy bão tại thời điểm cơn bão Usagi ngày 19-09-2013 lúc $00 \mathrm{Z}$ sẽ đưa vào đồng hóa với hệ thống WRF-LETKF (Phần 3).
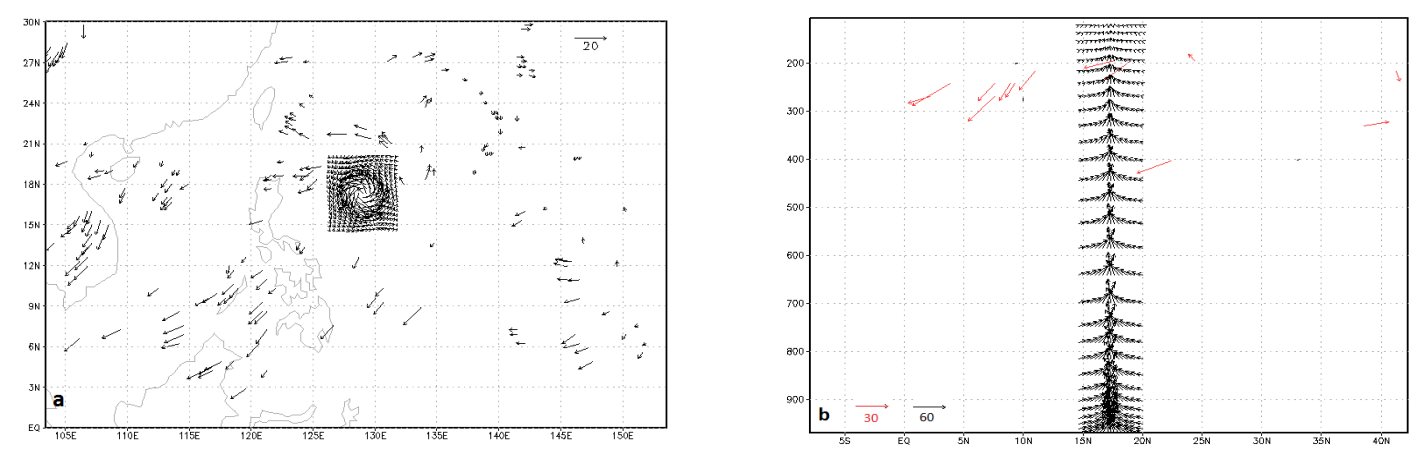

Hình 2. Minh họa gió quan trắc đồng thời giữa trường quy mô lớn AMV và xoáy nhân tạo tại mực $195 \mathrm{hPa}$ (hình $\mathrm{a}$ ) và gió mặt cắt tại kinh tuyến $130 \mathrm{E}$ (hình $\mathrm{b}$ ) của mô hình $\mathrm{WRF}-\mathrm{ARW}$ vào thời điểm $00 \mathrm{Z}$ ngày 19-09-2013. Đơn vị là $\mathrm{m} / \mathrm{s}$, độ dài véc tơ đại diện trong hình a là $20 \mathrm{~m} / \mathrm{s}$, trong hình $\mathrm{b}$ là $60 \mathrm{~m} / \mathrm{s}$ với gió cấu trúc bão (màu đen) và $30 \mathrm{~m} / \mathrm{s}$ với gió quy mô lớn (màu đỏ).

a)

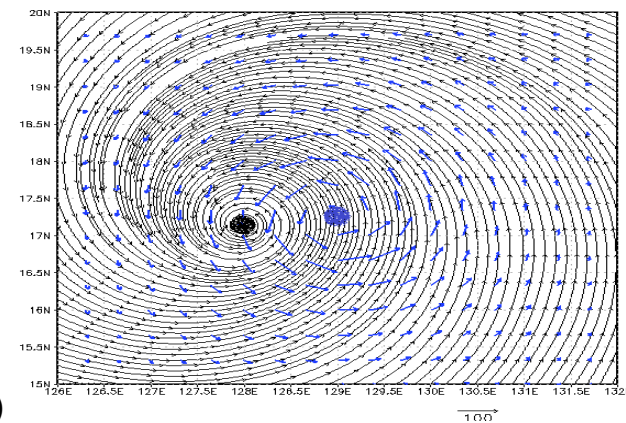

b)

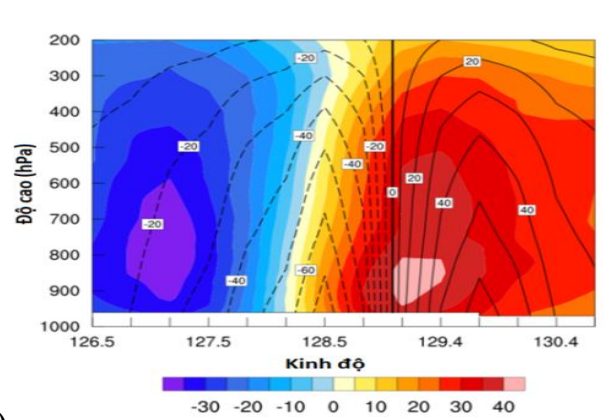

Hình 3. Trường gió bề mặt ban đầu của mô hình WRF-ARW (đường dòng) và của gió quan trắc nhân tạo (véc tơ gió màu xanh) tại vùng tâm bão (hình $\mathrm{a}$ ) và mặt cắt của thành phần gió tiếp tuyến giữa quan trắc nhân tạo (đường đồng mức liền) và ban đầu của mô hình (đường đẳng trị có tô màu) (hình $b$ ), minh họa cho cơn bão Usagi vào thời điểm $00 \mathrm{Z}$ ngày 19-09-2013. 
Tiếp theo trong hình $3 \mathrm{a}$ cho thấy sự khác biệt về vị trí tâm bão ban đầu của mô hình WRF-ARW khi sử dụng biên từ GFS và vị trị tâm bão nhân tạo theo phân tích quan trắc bão của JTWC. Tâm bão theo trường nền ban đầu của mô hình WRF-ARW có vị trí khoảng $17.0 \mathrm{~N} ; 128.0 \mathrm{E}$ trong khi theo tâm phân tích của JTWC là $16.9 \mathrm{~N} ; 128.5 \mathrm{E}$ (tâm của mô hình có xu thế lệch về phía Tây Tây Nam so với quan trắc).

\section{Thử nghiệm}

\subsection{Mô hình khu vưc WRF-ARW và hệ thống đồng hóa tổ hợ Kalman - LETKF}

Hệ thống mô hình khu vực WRF nhân động lực ARW phiên bản 3.2 (viết tắt là WRF-ARW) được sử dụng làm công cụ hạ quy mô động lực từ trường dự báo quy mô lớn của các mô hình toàn cầu. Trong nghiên cứu, hệ thống WRFARW được áp dụng với ba lưới lồng tương ứng với độ phân giải là $36 \mathrm{~km}, 12 \mathrm{~km}$ và $4 \mathrm{~km}$ trong đó các lưới độ phân giải $12 \mathrm{~km}$ và $4 \mathrm{~km}$ được thiết lập dịch chuyển theo tâm bão. Hệ thống đồng hóa số liệu sử dụng trong nghiên cứu là phương pháp lọc Kalman chuyển dạng tổ hợp địa phương LETKF phát triển cho mô hình WRF-ARW. Chi tiết hơn về hệ thống WRFARW và $L E T K F$ (gọi tắt là hệ thống WRFLETKF) do TS. Kiều Quốc Chánh phát triển có thể tham khảo trong [1].

Số liệu điều kiện biên được sử dụng là dự báo từ mô hình toàn cầu GFS (NCEP) hạn đến 120h cập nhật 6 tiếng một. Hai trường hợp thử nghiệm gồm thử nghiệm chuẩn CTRL với 21 thành phần với cấu hình vật lý khác nhau và trường phân tích ban đầu cho từng thành phần được sinh nhiễu trên nền GFS. Trường hợp thứ hai là $\mathrm{DABV}$ với cấu hình giống trường hợp CTRL nhưng có đồng hóa thông tin quan trắc hòa hợp giữa quan trắc quy mô lớn và xoáy nhân tạo bằng hệ LETKF. Hạn dự báo đến $120 \mathrm{~h}$ cho 4 ốp dự báo từ ngày 18/09/2013 đến 20/09/2013 cách nhau 12 tiếng một.

\subsection{Kết quả thư nghiệm cho con bão Usagi năm 2013}

\section{Ảnh hưởng đến truờng ban đầu}

Đối với số liệu quan trắc quy mô lớn từ quan trắc gió vệ tinh, khoảng $70-80 \%$ số nằm trên mực 400-300 hPa xuất phát từ đặc tính xác định của loại số liệu này thông qua các phần tử mây mỏng tựa trong (semi-transparent) trên cao [10]. Dưới mực $400 \mathrm{hPa}$, các thông tin chủ yếu liên quan đến cấu trúc bão và lấy từ số liệu xoáy nhân tạo TCVital. Số liệu AMV ít có ảnh hưởng phía trong bão những vẫn có thể giúp tăng cường thông tin từ các vùng lân cân bão. Thông qua số liệu quan trắc ở các mực cao, cấu trúc dòng phân kì ra ở các mực trên cao sẽ được tái cấu trúc lại và cũng giúp ích trong việc giảm thiểu thời gian thích ứng cho bão trong mô hình. Ngoài ra, việc hiệu chỉnh các dòng nền sẽ giúp cải thiện chất lượng dự báo quỹ đạo lên rất nhiều và cũng là cơ sở tạo ra các môi trường thực cho việc mô phỏng và dự báo thay đồi cường độ bão.

Trước hết ta xem xét hình 4 minh họa lại trường gió mực trên cao tại thời điểm phân tích của cơn bão Usagi lúc $00 z$ ngày 19/9/2013 trong đó vectơ màu đỏ là gió của mô hình GFS sẽ được sử dụng làm đầu vào cho mô hình WRF-ARW. Ta thấy ngay phía Đông Bắc (vĩ độ $\sim 22 \mathrm{~N}-24 \mathrm{~N}$ ) của cơn bão Usagi gió của GFS về hướng khá phù hợp với quan trắc nhưng cường độ có phần mạnh hơn. Như vậy khi thực hiện đồng hóa số liệu, về cơ bản gió khu vực phía Bắc cơn bão sẽ giữ nguyên hướng nhưng giảm đi về mặt độ lớn (thể hiện bằng các véc tơ gia số quan trắc và véc tơ gia số phân tích trong hình 5 , phải).

Hình 5 đưa ra mặt cắt ngang của véctơ gia số gió $^{1}$ ở mực 500 và $200 \mathrm{hPa}$. cho cơn bão Usagi để thấy được hiệu ứng của phương pháp đồng

\footnotetext{
${ }^{1}$ Increment vector, là véctơ gia số gió với hai thành phần là gia số cho thành phần gió $\mathrm{u}$ và gia số cho thành phần gió $\mathrm{v}$, gọi tắt là véctơ gia số. Ví dụ nếu vecto gió trường nền ban đầu là $\vec{V}^{\mathrm{b}}\left(\mathrm{u}_{\mathrm{b}}, \mathrm{v}_{\mathrm{b}}\right)$ và vecto quan trắc $\vec{V}^{\mathrm{o}}\left(\mathrm{u}_{\mathrm{o}}, \mathrm{v}_{\mathrm{o}}\right)$ thì vecto gia số quan trắc là hiệu của hai vecto này $\delta \vec{V}^{\mathrm{ob}}\left(\mathrm{u}_{\mathrm{o}}-\mathrm{u}_{\mathrm{b}}, \mathrm{v}_{\mathrm{o}}-\mathrm{v}_{\mathrm{b}}\right)$. Tương tự với $\vec{V}^{\mathrm{a}}\left(\mathrm{u}_{\mathrm{a}}, \mathrm{v}_{\mathrm{a}}\right)$ là vecto gió phân tích tối ưu cuối cùng thì vec tơ gia số phân tích là $\delta \vec{V}^{\mathrm{ab}}\left(\mathrm{u}_{\mathrm{a}}-\mathrm{u}_{\mathrm{b}}, \mathrm{v}_{\mathrm{a}}-\mathrm{v}_{\mathrm{b}}\right)$.
} 
hóa. Mực dưới (Hình 5, trái) hầu như chỉ có sự hiệu chỉ do số liệu bão nhân tạo trong khi mực trên cao có hiệu chỉnh cả quan trắc $\mathrm{AMV}$ ngoài hoàn lưu bão và bão nhân tạo (Hình 5 , phải). Lưu ý rằng thông tin nhân tạo từ TCVital cung cấp cấu trúc đến mực $300 \mathrm{hPa}$ và tập trung trong phạm vi cơn bão trong khi số liệu AMV bổ sung phía ngoài cơn bão và phía trên mực 300 hPa rất nhiều, hay nói cách khác, việc hòa hợp dữ liệu quan trắc cho phép tự bổ sung những khiếm khuyết của hai nguồn số liệu khác nhau khi đưa vào tăng cường chất lượng dự báo. Mặc dù vẫn còn tồn tại trong việc tái cấu trúc theo phương thẳng đứng nhưng cách tiếp cận này sẽ cho phép xem xét ảnh hưởng của việc đồng hóa cấu trúc bão và quy mô lớn tới các sai số dự báo quỹ đạo và cường độ cùng các đặc trưng khác của hệ thống dự báo tổ hợp (như trung bình tổ hợp hay độ tán của hệ thống tổ hợp) (Hình 4).

Ảnh hưởng đến sai số dụ báo quỹ đạo và cuoờng độ bão

Hình 6a,b cho thấy, đối với quỹ đạo dự báo cơn bão Usagi (2013) trong trường hợp CTRL

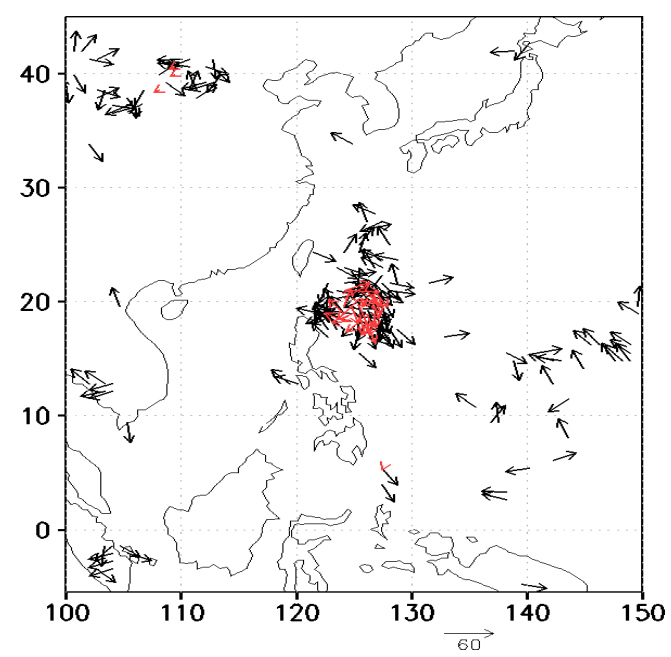

có $\mathrm{xu}$ thế dự báo lệch bắc cho các hạn trước 72h. Sau khi đồng hóa, trường hợp DABV đã cải thiện được sai số quỹ đạo ở hầu hết các hạn dự báo còn sai số cường độ được giảm chủ yếu tại các hạn $48 \mathrm{~h}$ và $72 \mathrm{~h}$.

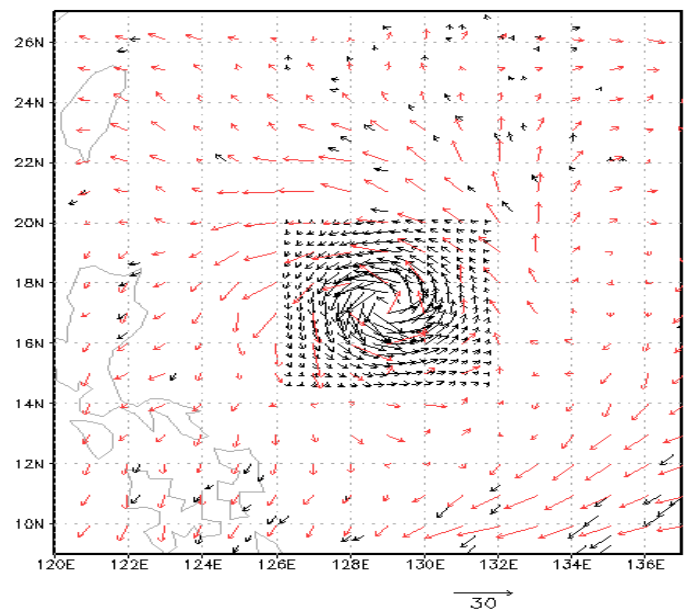

Hình 4. Trường gió mực $300 \mathrm{hPa}$ của GFS tại thời điểm ban đầu (véc tơ màu đỏ) và quan trắc gió $\mathrm{AMV}$ cùng xoáy nhân tạo (véc tơ màu đen). Vectơ tham khảo độ lớn có giá trị là $30 \mathrm{~m} / \mathrm{s}$.

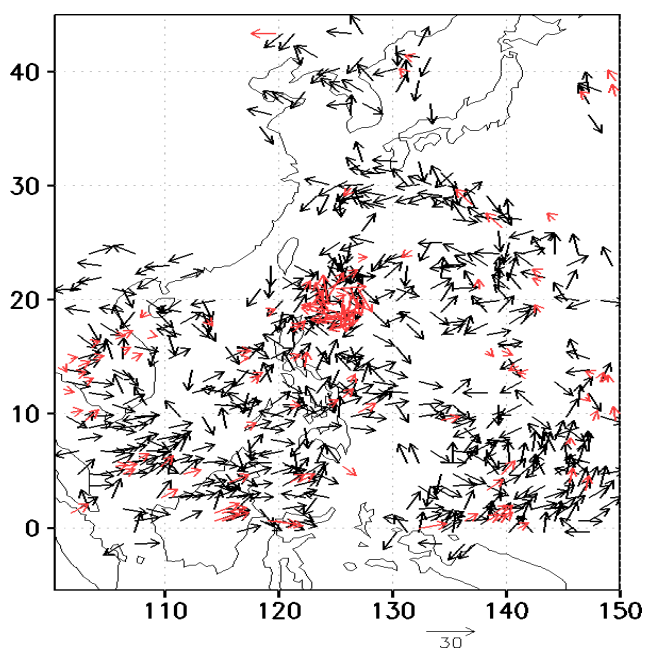

Hình 5. So sánh giữa véc tơ gia số quan trắc gió AMV kết hợp với TCVital (màu đỏ) và véc tơ gia số phân tích (màu đen) trong thử nghiệm $\mathrm{DABV}$ tại các mực $500 \mathrm{hPa}$ (trái) và $200 \mathrm{hPa}$ (phải) trên toàn bộ miền tính ngoài cùng $(36 \mathrm{~km})$ cho cơn bão Usagi lúc $00 \mathrm{Z}$ ngày $20 / 9 / 2013$. Vec tơ tham khảo độ lớn có giá trị là $60 \mathrm{~m} / \mathrm{s}$ cho mực $500 \mathrm{hPa}$ và $30 \mathrm{~m} / \mathrm{s}$ cho mực $200 \mathrm{hPa}$. 
a)

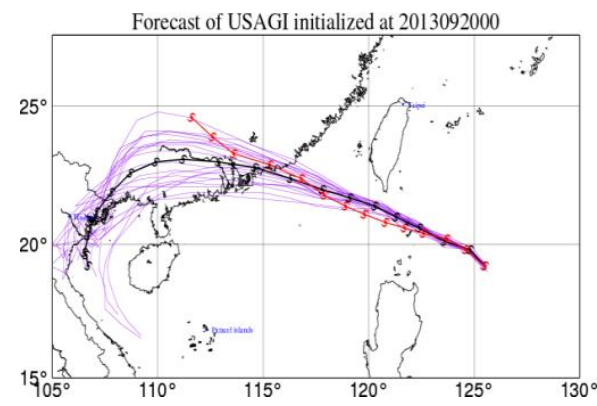

c)

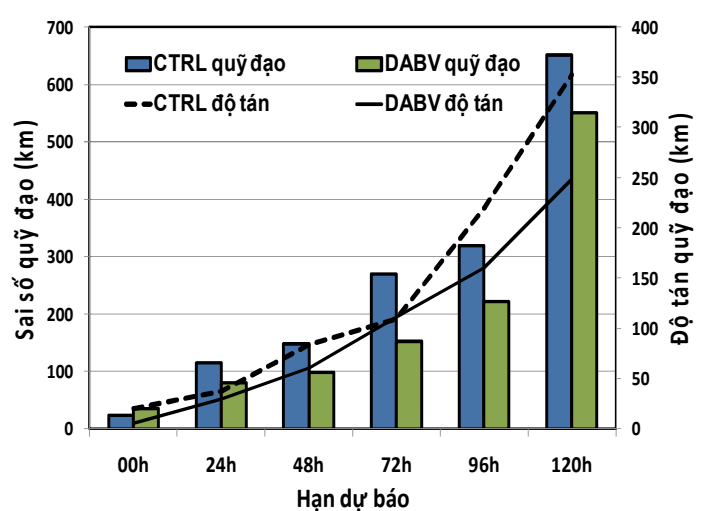

b)

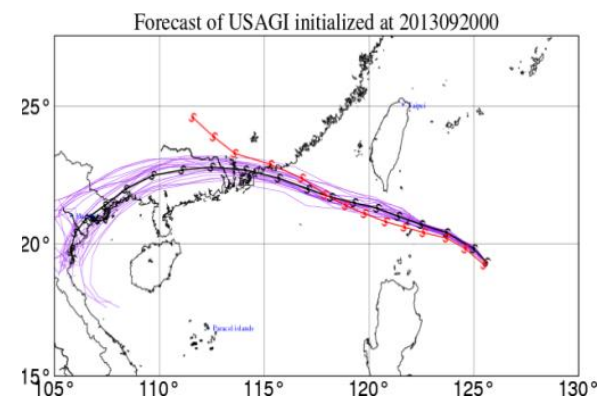

d)

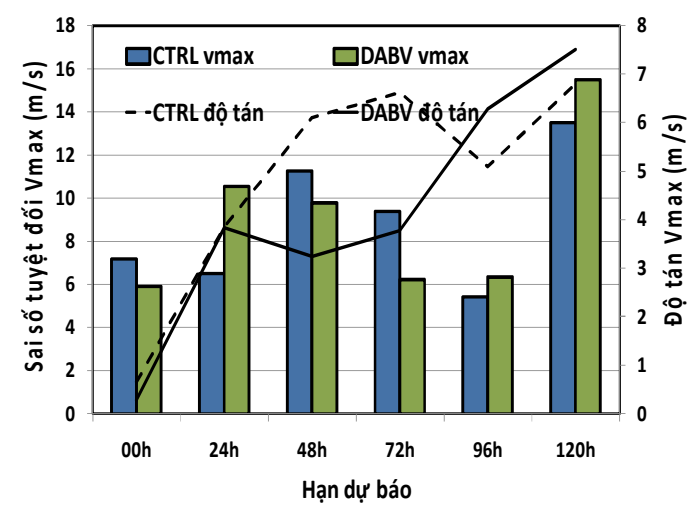

Hình 6. Minh họa quỹ đạo và cường độ dự báo cơn bão Usagi tại ốp 00z ngày 20-09-2013 của hai trường hợp CTRL (a) và $\mathrm{DABV}$ (b) (đường đỏ là quỹ đạo chuẩn, đường đen là trung bình tổ hợp và đường tím mảnh là quỹ đạo của từng thành phần tổ hợp); trung bình sai số từ tất cả các ốp dự báo cơn bão Usagi của quỹ đạo cùng độ tán tương ứng (c) và cường độ cùng độ tán tương ứng (d).

Điểm lưu ý đối với cơn bão Usagi là sau khi đi vào Biển Đông, cơn bão tiếp tục tăng cường độ trở lại ở thời điểm quanh các hạn dự báo 48h - 72h (ngày 22/09/2013) - các thời điểm mà sai số cường độ đã được giảm đi trong thử nghiệm DABV so với CTRL. Đối với vị trí ban đầu, mặc dù có sự hiệu chỉnh tốt ở một số chu kì dự báo nhưng về mặt trung bình các chu kì dự báo, sự khác biệt giữa $\mathrm{DABV}$ và $\mathrm{CTRL}$ là không đáng kể. Riêng cường độ ban đầu, việc đồng hóa thông tin xoáy trong trường hợp $\mathrm{DABV}$ đã giảm được sai số cường độ so với CTRL. Như vậy về cơ bản, trong 4 chu kì dự báo thử nghiệm cho cơn bão Usagi, trường hợp $\mathrm{DABV}$ giảm được sai số quỹ đạo trong hạn hầu hết các hạn dự báo nhưng sai số cường độ chỉ giảm đồng thời theo tại hai hạn dự báo $48 \mathrm{~h}$ và $72 \mathrm{~h}$. Về độ tán của mô hình, đối với quỹ đạo ít có sự khác biết giữa hai trường hợp ở hạn trước $96 \mathrm{~h}$. Đối với độ tán của dự báo cường độ, trong hạn dự báo từ $24 \mathrm{~h}-72 \mathrm{~h}$, trường hợp $\mathrm{DABV}$ nhỏ hơn CTRL kèm theo sai số thấp hơn trong hạn dự báo $48 \mathrm{~h}$ đến $72 \mathrm{~h}$ cho thấy ở hai hạn này tỉ lệ tin cậy của dự báo cường độ $\mathrm{DABV}$ tăng lên so với CTRL.

\section{Kết luận}

Bài báo đã giới thiệu phương pháp ban đầu hóa xoáy một cách khách quan khi sử dụng sơ đồ đồng hóa số liệu (ở đây là phương pháp LETKF) với quan trắc được thiết lập một cách đồng thời từ quan trắc quy mô lớn (số liệu gió vệ tinh CIMSS) và quan trắc quy mô bão (từ mô hình xoáy 3 chiều với đầu vào là các phân tích bão trong thực tế) và đã thử nghiệm trên mô hình khu vực WRF-ARW cho dự báo cơn 
bão Usagi hoạt động trên khu vực TBTBD năm 2013. Việc đưa các thông tin quan trắc vào đã cho thấy khả năng tăng cường thông tin về vị trí, cường độ ban đầu của xoáy bão kèm theo việc cải thiện dự báo chất lượng quỹ đạo ở hầu hết các hạn dự báo cho trường hợp thử nghiệm. Sai số dự báo cường độ được giảm chủ yếu ở hạn từ $48 \mathrm{~h}-72 \mathrm{~h}$ liên quan đến thời kì mạnh nhất của cơn bão và đồng thời sau khi đi vào Biển Đông. Việc đưa thông tin một cách khách quan, linh hoạt thông qua phương pháp đồng hóa tổ hợp LETKF đã cho phép bước đầu đưa được các thông tin phân tích bão trong nghiệp vụ vào bài toán dự báo xoáy thuận nhiệt đới cho các mô hình phân giải cao mà không cần thông qua các bước can thiệp vào trường ban đầu của mô hình như các phương pháp ban đầu hóa xoáy bão khác. Ngoài ra, các hiệu ứng của quá trình đồng hóa đồng thời hai loại thông tin ở hai quy mô khác nhau còn mang tính phi tuyến và cần thiết có những khảo sát trên tập mẫu lớn hơn.

\section{Tài liệu tham khảo}

[1] Kiều Quốc Chánh, (2011), "Xây dựng hệ thống đồng hóa lọc Kalman tổ hợp địa phương cho mô hình dự báo thời tiết WRF", Tạp chí Khoa học ĐHQGHN, Khoa học Tự nhiên và Công nghệ, Tập 27, (1S), tr. 17-28.

[2] Phan Văn Tân, Nguyễn Lê Dũng (2009), "Thử nghiệm ứng dụng hệ thống WRF-VAR kết hợp với sơ đồ ban đầu hóa xoáy vào dự báo quĩ đạo bão trên Biển Đông", Tạp chí Khí tượng Thủy văn, (583), tr. 1-9.

[3] Barbour, A.D., L. Holst, S. Janson (1993), "Poisson Approximation", Ann. Probab., 21 (4), pp. 2269-2279.
[4] Holland, G. (2008), "A revised hurricane pressure-wind model", Mon. Wea. Rev., (136), pp. 3432-3445.

[5] Holton, J.R. (2004), Introduction to Dynamic Meteorology, Elsevier Academic Press

[6] Kalnay, E., L. Hong, T. Miyoshi, S-C. Yang, and J. Ballabrera-Poy (2007), "4D-var or ensemble Kalman filter?", Tellus, (59A), pp. 758-773.

[7] Kieu, C. Q., and D.-L. Zhang, (2009), "An analytical model for the rapid intensification of tropical cyclones", Q. J. R. Meteor. Soc., (135), pp. 1336-1349.

[8] Kurihara, Y., M. A. Bender, and R. J. Ross (1993), "An initialization scheme of hurricane models by vortex specification", Mon. Wea. Rev., (121), pp. 2030-2045.

[9] Nguyen, H.V., and Y.L. Chen (2011), "High resolution initialization and simulations of typhoon Morakot (2009)", Mon. Wea. Rev., (139), pp. 1463-1491.

[10] Soden, B. J., C. Velden, and R. Tuleya (2001), "The Impact of Satellite Winds on Experimental GFDL Hurricane Model Forecasts", Mon. Wea. Rev., (129), 835-852.

[11] Tallapragada, V., and Coauthors (2014), Significant Advances to the NCEP Operational HWRF Modeling System for Improved Hurricane Forecasts, The 31st Conference on Hurricanes and Tropical Meteorology, San Diego, CA, Amer. Meteor. Soc., 14D.1.

[12] Wang, M., M. Xue, K. Zhao, and J. Dong (2014), "Assimilation of T-TREC-Retrieved Winds from Single-Doppler Radar with an Ensemble Kalman Filter for the Forecast of Typhoon Jangmi (2008)", Mon. Wea. Rev., (142), pp. 1892-1907.

[13] Zhang, M., Milija Zupanski, Min-Jeong Kim, and John A. Knaff (2013), "Assimilating AMSU-A Radiances in the TC Core Area with NOAA Operational HWRF (2011) and a Hybrid Data Assimilation System: Danielle (2010)", Mon. Wea. Rev., (141), pp. 3889-3907.

[14] Zou, X. and Q. Xiao (2000), "Studies on the Initialization and Simulation of a Mature Hurricane Using a Variational Bogus Data Assimilation Scheme", J. Atmos. Sci., (57), pp. 836-860. 


\title{
Improving Initial Tropical Cyclone Conditions in Numerical Weather Prediction with Blending Storm-scale and Large-Scale Observations
}

\author{
Du Duc Tien ${ }^{1}$, Ngo Duc Thanh ${ }^{2}$, Kieu Quoc Chanh ${ }^{3}$ \\ ${ }^{I}$ National Center for Hydro-Meteorological Forecasting, Hanoi, Vietnam \\ ${ }^{2}$ University of Science and Technology of Hanoi, Vietnam Academy of Science and Technology, Vietnam \\ ${ }^{3}$ Indiana University, Indiana, USA
}

\begin{abstract}
Using blending observations from storm-scale to large-scale, the study present an approach for initializing tropical cyclone conditions in numerical weather prediction models WRFARW developed by NCEP using the local ensemble transformed Kalman filter (LETKF) assimilation method. The storm-scale observation was created by using 3-dimension tropical cyclone model with real-time cyclone analysis parameters from NCEP (tropical cyclone vital reports) and the large-scale observation was created based on cloud motion wind of the Cooperative Institute for Meteorological Satellite Studies (University of Wisconsin). Some first experiments were carried out with severe tropical cyclone Usagi in the north Western Pacific (2013) and the results showed that this method can help increasing both track and intensity tropical cyclone forecast.
\end{abstract}

Keywords: Tropical cyclone initialization, 3-dimension tropical structure. 